\title{
Microbial composition of enigmatic bird parasites: Wolbachia and Spiroplasma are the most important bacterial associates of quill mites (Acari: Syringophilidae)
}

\author{
Eliza Glowska ${ }^{1, *}$, Zuzanna K. Filutowska ${ }^{2}$, Miroslawa Dabert ${ }^{2}$, Michael Gerth ${ }^{3}$ \\ 1 Adam Mickiewicz University in Poznań, Faculty of Biology, Department of Animal \\ Morphology, 61-614 Poznań, Poland \\ 2 Adam Mickiewicz University in Poznań, Faculty of Biology, Molecular Biology \\ Techniques Laboratory, 61-614 Poznań, Poland \\ 3 University of Liverpool, Institute for Integrative Biology, Biosciences Building, Crown \\ Street, L69 7ZB, Liverpool, United Kingdom \\ *glowska@amu.edu.pl
}

\begin{abstract}
The microbiome is an integral component of many animal species, potentially affecting behaviour, physiology, and other biological properties. Despite this importance, bacterial communities remain vastly understudied in many groups of invertebrates, including mites. Quill mites (Acariformes: Syringophilidae) are a poorly known group of permanent bird ectoparasites that occupy quills of feathers and feed on bird subcutaneous tissue and fluids. Most species have strongly female biased sex ratios and it was hypothesized that this is caused by endosymbiotic bacteria. Their peculiar lifestyle further makes them potential vectors for bird diseases. Previously, Anaplasma phagocytophilum and a high diversity of Wolbachia strains were detected in quill mites via targeted PCR screens. Here, we use an unbiased $16 \mathrm{~S}$ amplicon sequencing approach to determine other Bacteria that potentially impact quill mite biology.

We performed 16S V4 amplicon sequencing of 126 quill mite individuals from eleven species parasitizing twelve bird species (four families) of passeriform birds. In addition to Wolbachia, we found Spiroplasma as potential symbiont of quill mites. Interestingly, consistently high Spiroplasma titres were only found in individuals of two mite species associated with finches of the genus Carduelis, suggesting a history of horizontal transfers of Spiroplasma via the bird host. Furthermore, there was evidence for Spiroplasma negatively affecting Wolbachia titres. We found no evidence for the previously reported Anaplasma in quill mites, but detected the potential pathogens Brucella and Bartonella at low abundances. Other amplicon sequence variants (ASVs) could be assigned to a diverse number of bacterial taxa, including several that were previously isolated from bird skin. We observed a relatively uniform distribution of these ASVs across mite taxa and bird hosts, i.e, there was a lack of host-specificity for
\end{abstract}


most detected ASVs. Further, many frequently found ASVs were assigned to taxa that show a very broad distribution with no strong prior evidence for symbiotic association with animals. We interpret these findings as evidence for a scarcity or lack of resident microbial associates (other than inherited symbionts) in quill mites, or for abundances of these taxa below our detection threshold.

Keywords $16 \mathrm{~S}$ amplicon sequencing, Bartonella, birds, Brucella, ectoparasites, Ion Torrent, quill mites

\section{Introduction}

There is abundant evidence that microbial taxa are an essential component of many animal species [1]. Bacteria-encoded traits may significantly impact host phenotypes, e.g. through providing essential nutrients $[2,3]$, defending against pathogens $[4,5]$, but also affecting ecological features of their hosts, such as mate choice [6] and life history traits [7]. Because of their potential importance in understanding the biology of many organisms, the number of microbiome studies has been soaring [8]. This popularity is owed to methodological advances (high-throughput sequencing technologies) allowing comprehensive investigation of the microbial communities [9], but also to the decreasing costs of these approaches [10]. However, the main focus of microbiome studies so far has been vertebrates [11]; while in invertebrates, the focus has been on taxa of medical, veterinary, or economical importance. For example, in mites, microbiome studies have been conducted on the pests of stored food products [12, 13], dust mites producing allergenic agents [14-16], and mites transmitting pathogens, such as sheep scab mites [17] red poultry mites [18, 19], and the honey bee parasite Varroa [20].

In the present study, we have focussed on quill mites (Acariformes: Syringophilidae). These obligatory bird ectoparasites live and reproduce inside the quills of feathers where they feed on subcutaneous fluids such as lymph and blood. Quill mite dispersion has been observed on the same individual (from infected to uninfected feathers), between individuals of the same species (e.g., from parents to hatchings) and occasionally by transfer between gregarious bird species [21-24]. This mode of feeding and dispersion makes quill mites potential vectors for bacterial pathogens, similar to ticks or lice [25]. However, only two bacterial taxa were recorded in quill mites so far: 1) Anaplasma phagocytophilum (Alphaproteobacteria, Rickettsiales) was detected in two quill mite species from three bird species [26]; 2) Multiple genetically distinct lineages of Wolbachia (Alphaproteobacteria, Rickettsiales) were found in five species of quill mites [27]. As these studies were targeted PCR screens, it remains unclear what other Bacteria populate quill mites. Furthermore, the importance of quill mites for bird pathogen dynamics is not known.

To address these questions, we here assess the bacterial composition of 126 quill mite individuals encompassing eleven species with a more unbiased 16S rRNA amplicon sequencing approach. We find that the symbionts Wolbachia and Spiroplasma are 
among the most commonly taxa associated with quill mites. Other taxa include Bacteria that were previously found in association with arthropods, and Bacteria with a very broad distribution. Strikingly, neither quill mite taxonomy nor bird host taxonomy significantly influences bacterial composition in quill mites. Furthermore, we find that despite the detection of Bartonella and Brucella, quill mites do not seem to be a major pathogen vector in birds.

\section{Materials and methods}

Animal collection and DNA extraction. A summary of collected quill mite species and their bird hosts can be found in Table 1. All quill mites used in this study were collected in Kopan, Poland during spring migration of birds monitored by the Bird Migration Research Station, University of Gdansk, April 2009. One secondary flight feather was completely removed from each bird specimen and dissected under a stereo microscope (Olympus ZS30). Individual mites were washed twice and preserved in $96 \%$ ethanol and total genomic DNA was extracted from single specimens using DNeasy Blood \& Tissue Kit (Qiagen GmbH, Hilden, Germany) as described previously [28]. This procedure leaves the exoskeletons intact, and the specimens were subsequently mounted on microscopic slides in Faure medium, and determined using the key from Skoracki et al. (2016) [29]. All morphological observations were carried out with an Olympus BH2 microscope with differential interference contrast (DIC) optics and a camera lucida. All DNA samples and corresponding voucher specimens are deposited in the collection of the Department of Animal Morphology, Faculty of Biology, Adam Mickiewicz University in Poznań, Poland. To identify potential contaminants, in addition to sequencing a negative control alongside all samples, we further extracted DNA from reagents and materials commonly used in the laboratory this work was carried out in. One library each was created from extraction buffer (ALT), millipore water, microscope swabs, pipette swabs, and swabs of other equipment (pincettes, scalpels, benches, etc). These five libraries were processed and sequenced separately from the other samples, but by using identical procedures.

Library preparation and sequencing. We amplified and sequenced the V4 hypervariable region of $16 \mathrm{~S}$ rRNA gene. For the PCRs, each $10 \mu \mathrm{l}$ sample was prepared in two technical replicates containing $2 \mu \mathrm{l}$ HOT FIREPol Blend Master Mix (Solis

Biodyne, Tartu, Estonia), $0.25 \mu \mathrm{M}$ of each double-indexed fusion primer (Supplementary Table S1), and about $1 \mathrm{ng}$ of template DNA. The fusion PCR regime used was $12 \mathrm{~min}$ at $95^{\circ} \mathrm{C}, 40$ cycles of $15 \mathrm{sec}$ at $95^{\circ} \mathrm{C}, 30 \mathrm{sec}$ at $58^{\circ} \mathrm{C}, 30 \mathrm{sec}$ at $72^{\circ} \mathrm{C}$, and a final $7 \mathrm{~min}$ at $72{ }^{\circ} \mathrm{C}$. After PCR, all samples were pooled, size-selected on a $3 \%$ agarose gel, purified using the QIAquick Gel Extraction Kit (Qiagen), and quantified on a 2200 TapeStation (Agilent Technologies, Inc.). Clonal template amplification on Ion Sphere Particles (ISPs) was performed using the Ion Torrent One Touch System II and the Ion PGM ${ }^{\mathrm{TM}}$ Hi- $\mathrm{Q}^{\mathrm{TM}}$ View OT2 Kit with regard to manufacturer's instructions. Sequencing of the 
Table 1. Overview of quill mites sampled for this study.

\begin{tabular}{llc}
\hline Quill mite species & Bird host species (common name) & Number of individuals \\
\hline Aulobia cardueli & Carduelis flammea (Common redpoll) & 2 \\
Aulobia cardueli & Carduelis spinus (Eurasian siskin) & 4 \\
Syringophiloidus parapresentalis & Turdus iliacus (Redwing) & 3 \\
Syringophilopsis fringillae & Fringilla coelebs (Common chaffinch) & 6 \\
Syringophilopsis kirgizorum & Carduelis carduelis (European goldfinch) & 9 \\
Syringophilopsis kirgizorum & Carduelis chloris (European greenfinch) & 2 \\
Syringophilopsis turdi & Turdus iliacus (Redwing) & 15 \\
Syringophilopsis turdi & Turdus philomelos (Song thrush) & 4 \\
Torotrogla cardueli & Carduelis carduelis (European goldfinch) & 6 \\
Torotrogla cardueli & Carduelis spinus (Eurasian siskin) & 13 \\
Torotrogla gaudi & Fringilla coelebs (Common chaffinch) & 16 \\
Torotrogla lusciniae & Luscinia luscinia (Thrush nightingale) & 7 \\
Torotrogla lusciniae & Luscinia svecica (Bluethroat) & 1 \\
Torotrogla merulae & Turdus merula (Common blackbird) & 13 \\
Torotrogla merulae & Turdus philomelos (Song thrush) & 9 \\
Torotrogla modularis & Prunella modularis(Dunnock) & 4 \\
Torotrogla rubeculi & Erithacus rubecula (European robin) & 12 \\
\hline
\end{tabular}

templated ISPs was conducted on the Ion $318^{\mathrm{TM}}$ Chip with the use of Ion PGM ${ }^{\mathrm{TM}}$ Hi- $\mathrm{Q}^{\mathrm{TM}}$ View Sequencing Kit and Ion PGM system (Ion Torrent, Thermo Fisher Scientific, Inc.) at Molecular Biology Techniques Laboratory, Faculty of Biology, AMU. All reads resulting from the sequencing are available under NCBI BioProject accession PRJNA482380.

Read processing and statistical analyses. Reads were trimmed of adaptors and primer sites by using cutadapt version 1.16 [30]. The remaining reads were dereplicated, denoised, and chimeras eliminated using the DADA2 package version 1.8 [31] within the $\mathrm{R}$ statistical programming environment [32]. Taxonomic assignment of the ASVs (amplicon sequence variants), to species level where possible, was also performed within DADA2 using the SILVA database version 132 [33]. Next, contaminant taxa were identified from the sequenced extraction control using the 'prevalence' algorithm implemented in the R package decontam [34]. Further potential contaminants were identified by processing the five libraries derived from reagents and materials as described, and then excluding all ASVs that were found in any of these control libraries from subsequent analyses.

To reduce the impact of ASVs with very low abundance, we removed all ASVs that were present in only a single sample and also discarded ASVs from Bacterial Phyla that only occurred once in total. To account for potential biases between samples with uneven sequencing depth, all read counts from the remaining samples were rarefied to the read depth of the sample with the lowest read number. An overview of how our filtering steps affected ASV counts can be found in Supplementary Table S2. All subsequent statistical analyses were done on log-transformed read counts. Because the symbionts Wolbachia and Spiroplasma were dominant in some of the samples, we excluded all ASVs corresponding to these taxa prior to statistical comparisons between 
groups. First, we plotted the abundance of the most frequently found bacterial families using the $\mathrm{R}$ packages phyloseq and ggplot2 [35, 36]. Next, ordination analyses were performed with phyloseq using Bray distances and non-metric multidimensional scaling (NMDS). Differences in abundances of particular taxa between groups (quill mite species, bird host species, developmental stage, Wolbachia positive and negative samples) were determined with Kruskal-Wallis rank sum tests, and p-values were adjusted to these multiple comparisons to control for the false discovery rate [37]. These tests were done separately for differences in abundance of bacterial phyla, orders, families, and genera. Furthermore, we calculated Jensen-Shannon distances between the aforementioned groups and used adonis tests (analysis of variance using distance matrices) implemented in the $\mathrm{R}$ package vegan [38] to test if they differed significantly. The phyloseq object file containing all data used in the described analyses is available as Additional file 1.

\section{Results}

We have investigated the microbial composition of 126 individuals belonging to eleven quill mite species that parasititze twelve bird host species of passeriform birds.

Amplicon sequencing of the v4 region of the $16 \mathrm{~S}$ rRNA gene on an IonTorrent resulted in $1,582,340$ reads, with 9,426 reads per sample on average $(4,616-20,231)$. After

97

98 99 100 101 102 103 104
Spiroplasma Wolbachia Other a)

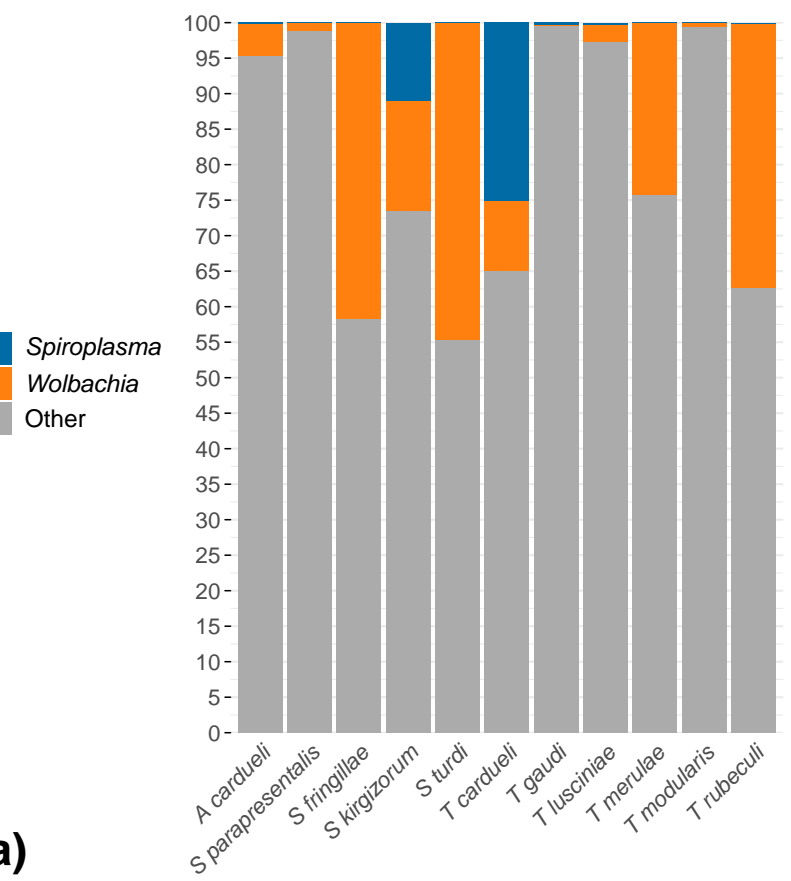

Actinomycetaceae Burkholderiaceae Chitinophagaceae

Corynebacteriaceae

Enterobacteriaceae

Hymenobacteraceae

Microbacteriaceae

Micrococcaceae

Moraxellaceae

Mycobacteriaceae

Pasteurellaceae

Pseudomonadaceae

Rhizobiaceae

Rhodobacteraceae

Sphingomonadaceae

Staphylococcaceae

Streptococcaceae

Weeksellaceae

Xanthobacteraceae

Xanthomonadaceae

b)

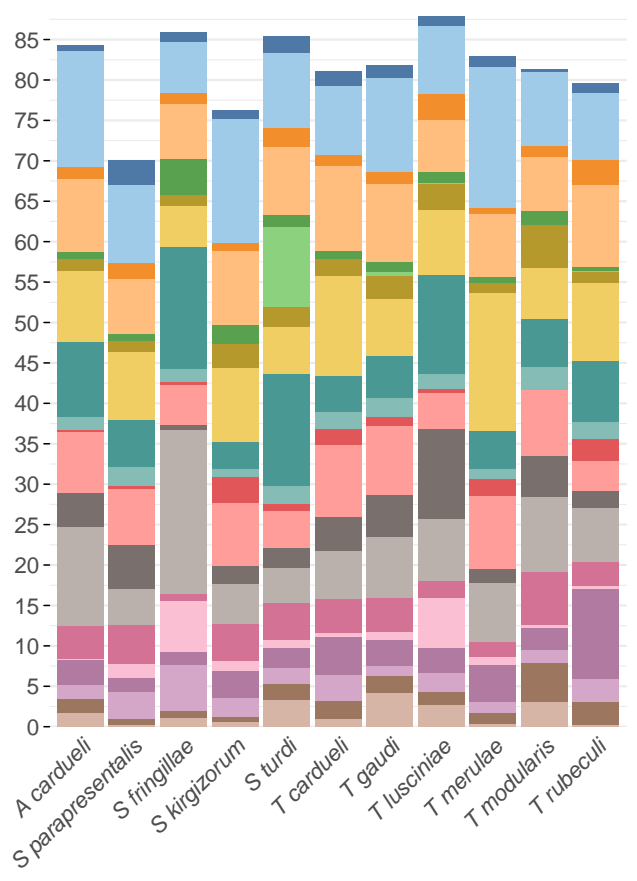

Figure 1. Overview of the bacterial taxa detected in quill mites. a) Relative abundances for the endosymbionts Spiroplasma and Wolbachia. b) Relative proportions of the 20 most abundantly found bacterial families in a dataset without the symbionts Spiroplasma and Wolbachia. For $\mathbf{a}$ ) and b), each bar represents the averaged abundances across all samples of a single species. Height of stacks represent relative abundances of each Bacterial taxon. For abundance plots of all samples, please refer to Supplementary Figure S1. 
Figure 2. Similarity of quill mite microbiota without the endosymbionts Spiroplasma and Wolbachia. Ordination analysis is based on non-metric multidimensional scaling (NMDS) and bray distances. Log-transformed abundances were analysed. Colors of the dots represent different quill mite species from which the samples were isolated. Shape of the dots represent for $\mathrm{Wol}_{\text {- }}$ bachia infection status. processing of reads (quality filtering, denoising, annotation, low-abundance filtering, rarefying, decontamination- see Materials and methods for details), 912 ASVs were retained. Among the most abundant bacterial taxa found in quill mites were Wolbachia and Spiroplasma (Fig, 1a, Supplementary Figure S1), both of which are vertically transmitted symbionts associated with a broad range of arthropods. Because these symbionts were not equally abundant across samples and might thus bias estimates of bacterial composition, they were excluded from the subsequent analyses.

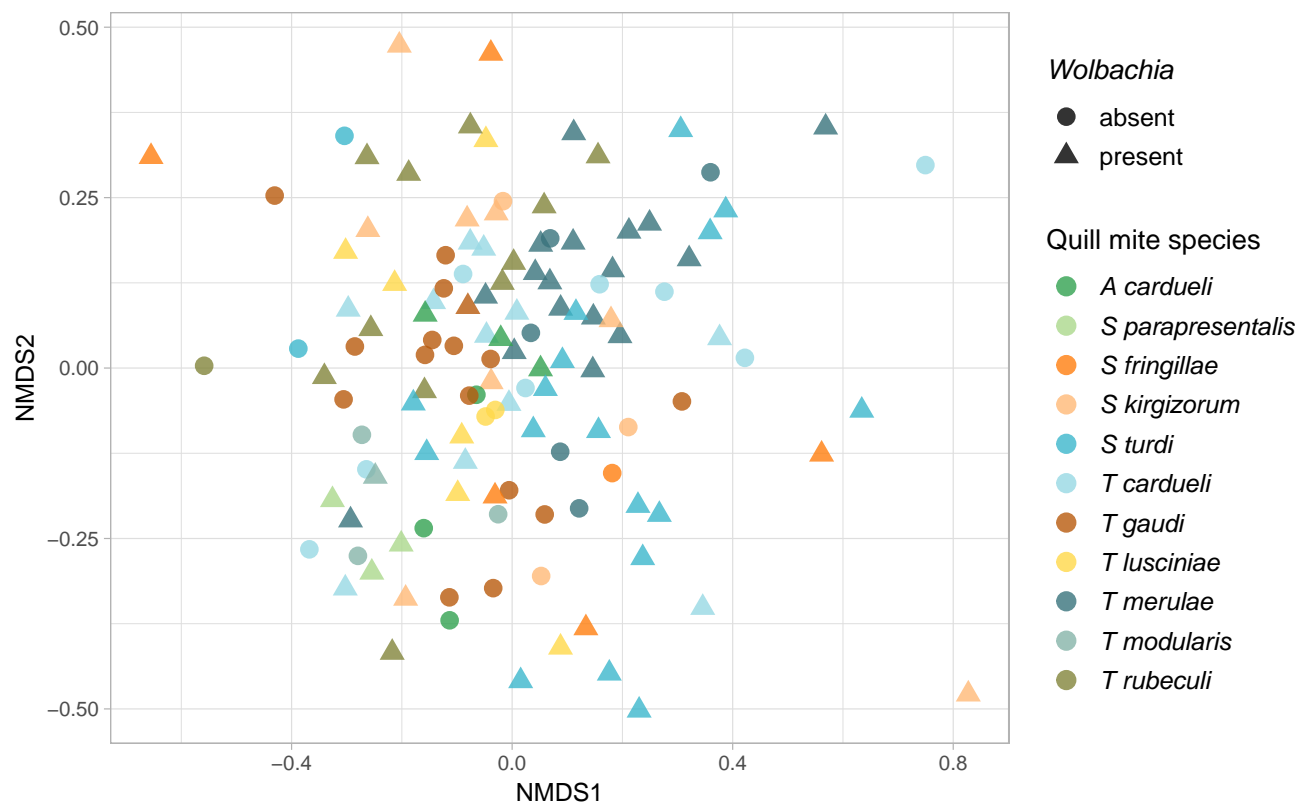

Bar plots of ASV abundance and ordination analyses with this filtered dataset revealed that the bacterial composition was relatively uniform across samples, and no clear differentiation between samples extracted from different mite species, or between Wolbachia positive and negative samples could be observed (Figs. 1b \& 2, see also Supplementary Figure S1). However, when trying to identify differential abundance patterns of microbial composition between groups using analysis of variances, we found that bacterial composition was more similar between samples from the same quill mite species or genus and bird host species or genus than expected by chance. Furthermore, six bacterial families were found to be differentially abundant between quill mite species with a Kruskal-Wallis test ( $<<0.01$, Fig. 3), one of which (Xanthobacteraceae) was also found to differ between samples of different bird host genera.

Out of 912 detected ASVs, the ten most abundantly encountered genera were Micrococcus, Corynebacterium, Acinetobacter, Streptococcus, Burkholderia, Phyllobacterium, Ralstonia, Mycobacterium, Paracoccus, and Sediminibacterium (see Supplementary Table S2 for a full list of ASVs). None of these taxa seemed dominant in any sampled group (based on mite or bird taxonomy), and the 20 most abundant families made up similar proportions of the total ASVs across samples (Fig. 1b, 


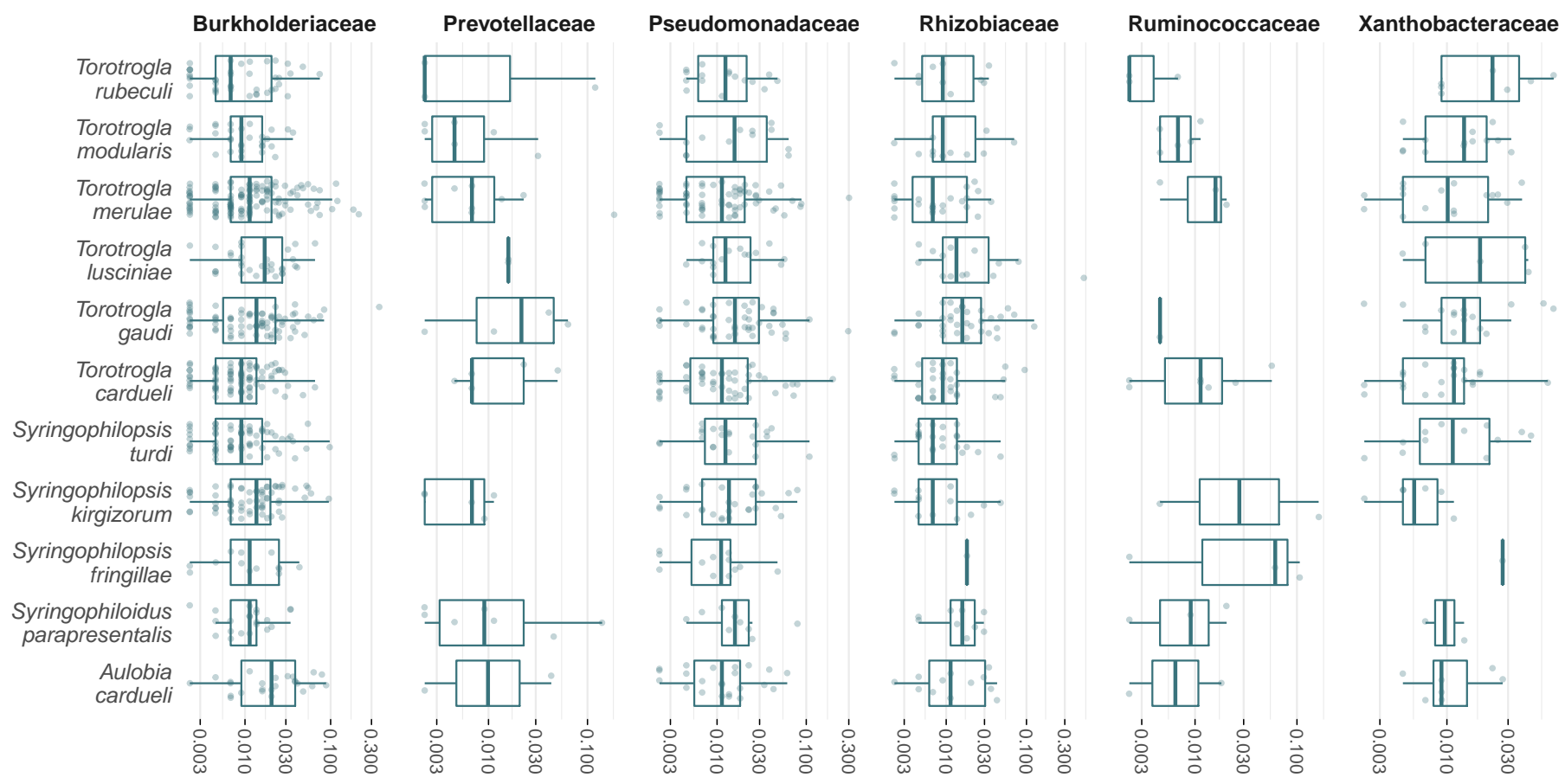

Figure 3. Abundance of five bacterial families that were found to be differentially abundant between quill mite species analysed. Counts for the symbionts Wolbachia and Spiroplasma were excluded.

As opposed to the general trend in the microbiome composition data, there was strong evidence for differential abundance of the symbionts Wolbachia and Spiroplasma between the bird hosts from which the mites were collected. For example, high Spiroplasma titres were only observed in two mite species collected from the host genus Carduelis (Fig. 4a, Supplementary Table S4). Further, although Wolbachia was present in mites sampled from all bird hosts, it was especially prevalent in mites collected from Turdus sp., Erithacus sp., and Fringilla sp. In contrast, it was absent or at very low titres in mites parasitizing Luscinia sp. (Fig. 4a). On average, the abundance of Wolbachia was lower in samples that also contained Spiroplasma (Figs. 1a, 4b). Notably, this was not an effect of Spiroplasma presence reducing the amount of available reads for Wolbachia (Fig. 4b). For mites harbouring both symbionts (eleven samples in total), we found that the abundances for Wolbachia and Spiroplasma seemed to be positively correlated (Fig. 4c). 
Figure 4. Relative abundances of the endosymbionts Wolbachia and Spiroplasma in quill mites. a) Abundances for all samples that are Spiroplasma and/or Wolbachia positive, sorted by bird host species from which the quill mites were isolated. Bird species phylogeny was taken from Jetz et al. ([39]; https://birdtree.org/). b) Relative Wolbachia abundances in samples with and without Spiroplasma. c) Correlation of Wolbachia and Spiroplasma abundances for samples in which both symbionts were present. For b) and c), only samples with abundances $\geq 1 \%$ are shown. Also, to avoid biases of abundance estimates based on a single dominant taxon, the counts were corrected for the presence of the other endosymbiont. For example, Wolbachia abundance here refers to the abundance ratio of Wolbachia vs all other non-Spiroplasma ASVs. For uncorrected Spiroplasma and Wolbachia abundances for all samples, please refer to Supplementary Table S3 and Table S4. a)

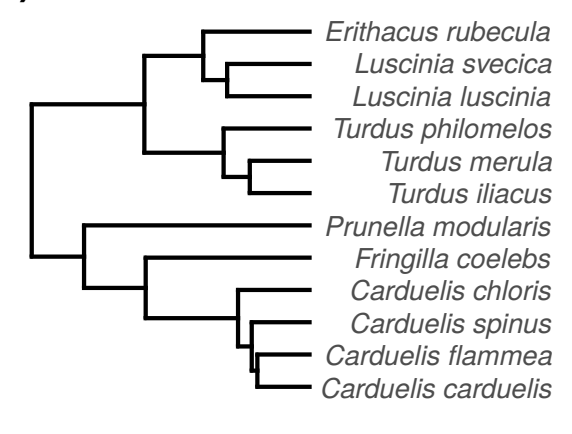

b)
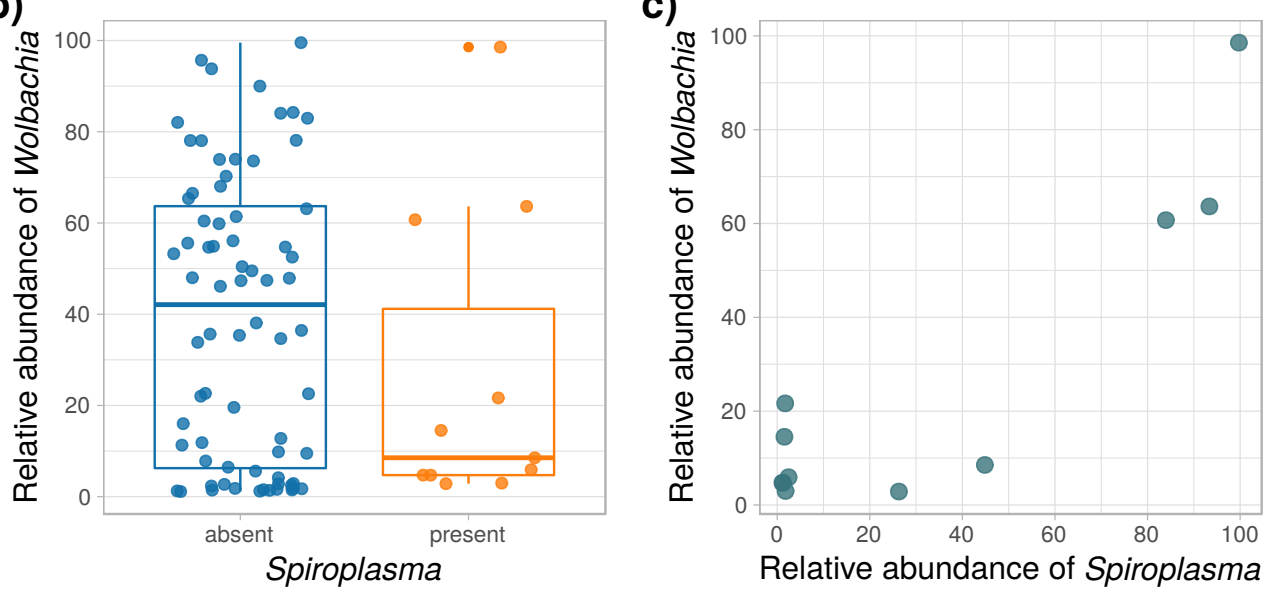

c)

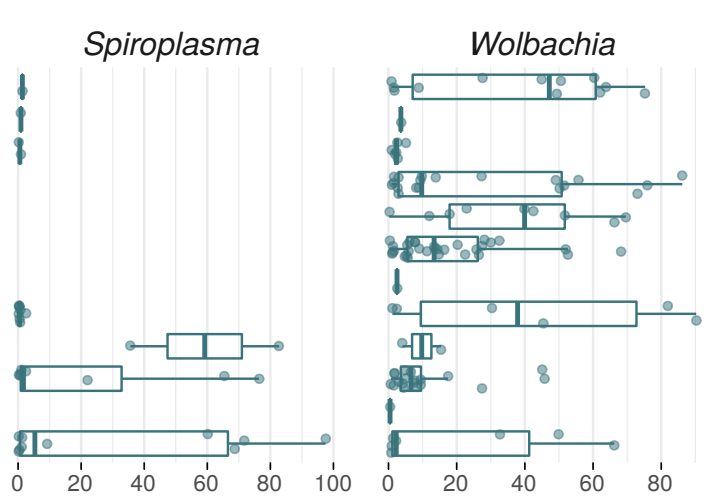

Relative abundance of Spiroplasma

\section{Discussion}

\section{Origin of microbial DNA in quill mites}

We here have sequenced microbial taxa from quill mites, an enigmatic group of bird ectoparasites. The taxa detected through $16 \mathrm{~S}$ sequencing may be 1) resident symbionts of quill mites, 2) environmentally acquired, transient bacteria, or 3) contaminants from reagents and materials. Each of these options comes with a number of assumptions that can be tested with our data.

1) For "true", resident symbionts, one would expect high abundances in at least some of the investigated hosts, presence in all individuals of a host species, and specialization of the symbionts, measurable as genetic differentiation between the symbionts of different host taxa. For example, all honey bees (Apis sp.) harbour seven core gut microbial taxa, five of which are present in other corbiculate bees, and two that are not found anywhere else [40]. The composition of these taxa is correlated with phylogenetic distances in this clade of bees, suggesting long-term association of the microbes with bees. In our dataset, Wolbachia and Spiroplasma are the most likely candidates for true symbiotic associations. Both Bacteria are known as endosymbionts 
from other arthropods, and are unable to permanently live outside their hosts [41, 42]. Further, we document a very high abundance of these taxa in at least some of the investigated samples (Fig. 4), which is in line with the assumptions above. In a previous study, Wolbachia strains of quill mites were investigated with a multi locus approach and it has been shown that quill mite associated strains are genetically very different to any other Wolbachia strains described so far [27]. Here, we have found 8 different ASVs annotated as Wolbachia, each of which is $100 \%$ identical to at least one Wolbachia $16 \mathrm{~S}$ sequence previously isolated from quill mites. For Spiroplasma, we found a single ASV that is only $92 \%$ identical to the next closest match in the Silva database. This implies that Spiroplasma in quill mites might be genetically distinct from Spiroplasma of other arthropods, as is the case for Wolbachia. However, sequencing data of more loci are needed to establish the phylogenetic placement of Spiroplasma from quill mites.

2) For environmentally acquired, transient taxa, the expectation is that the microbial composition detected in the host reflects the microbial composition of its environment stronger than it reflects host-specific factors. For example, the gut microbiome of some caterpillars is dominated by Bacteria that derive from their food, evidenced by similar bacterial composition of leave surfaces and caterpillar faeces [43]. Quill mites live permanently within feather quills of their bird hosts, hence one might expect to find similar taxa in feathers or on bird skin as in quill mites. Unfortunately, none of the bird hosts sampled in our study was investigated previously with regard to resident skin or feather microbes. One of the most comprehensive feather microbiome studies was performed in the Dark-eyed Juncos (Junco hyemalis) and revealed that feathers of these birds harbour bacteria commonly occurring in the soil and phyllosphere (Brevundimonas, Methylobacterium, Sphingomonas), as well as potential plant pathogens (e.g. Sphingomonas, Microbacterium, Curtobacterium, Rathayibacter) [44]. All of these taxa were also found in our study, suggesting a potential environmental determinant of the bacterial composition we observed in quill mites. Furthermore, many of the core bacterial families described in bird skin microbiome studies were also found in quill mites (e.g., Pseudomonadaceae, Methylobacteriaceae, Corynebacteriaceae, Moraxellaceae, Mycobacteriaceae, Leuconostocaceae, Staphylococcaceae, Lactobacillaceae, Micrococcaceae, Streptococcaceae, Enterobacteriaceae, Sphingomonadaceae, Neisseriaceae, Xanthomonadaceae and Weeksellaceae) [45, 46]. Despite these similarities, and some statistical support for bird hosts shaping the microbiome community in our study, the lack of clustering in ordination analysis indicates that environment is not the major determining factor of quill mite microbiome composition.

3) Importantly, contaminants from reagents and kits may significantly impact microbiome compostion estimates, especially when using low biomass samples such as quill mites [47-49]. This is problematic in any microbiome study, and is very difficult to exclude with certainty. Here, we removed contaminants statistically in silico based on the microbial composition of the sequenced extraction control [34]. Further, we removed all ASVs present in independently sequenced controls derived from reagents and 
equipment commonly used in the laboratory where this study was performed (see Materials and methods). However, a number of ASVs we recovered correspond to common kit contaminants in $16 \mathrm{~S}$ microbiome studies (e.g., Ralstonia, Kocuria), human skin Bacteria (Corynebacterium) or ubiquitous taxa with no strong evidence for symbiotic associations with arthropods (Pseudomonas, Acinetobacter). These taxa might constitute true associates, but we cannot exclude the possibility that they originate from contaminating sources.

In summary, we found a diverse range of Bacteria associated with quill mites. The lack of differentiation between different mite species or between species collected from different bird hosts leads us to conclude that there are no strong associations with typical gut bacteria as observed in other arthropods. However, we cannot exclude that we missed such potential associates due to the limited amount of DNA that can be extracted from the minute hosts.

\section{Exchange of Bacteria via bird hosts}

Due to their ectoparasitic lifestyle with occasional host switching, quill mites have the potential to transmit Bacteria between their hosts. Here, we detected two pathogenic microbes that might be important in that respect: Brucella and Bartonella. Brucella is the agent of brucellosis, which is considered one of the most widespread zoonotic infections [50]. Several Brucella species are a human health threat, and people typically become infected through contact with domesticated Brucella infected animals, such as goats, sheep, or swine [51]. However, several bloodsucking arthropods, such as ticks and lice are regarded as possible vectors for Brucella [52-54]. To our knowledge, there is no data indicating that Acari other than ticks are natural Brucella carriers. It was hypothesized that birds and other wild animals act as natural reservoirs for Brucella [55], which is in line with our finding of this Bacterium in bird ectoparasites. The importance of quill mites in spreading Brucella between bird species remains to be assessed, but it prevalence (20/126 investigated individuals, 8 different mite species) suggests this finding is of potential importance in understanding this pathogen's dynamics.

Bartonella are gram-negative Bacteria that are typically transmitted by blood sucking arthropods, and are infectious in mammalian hosts [56-58]. There are also reports on Bartonella incidence in birds [59,60], and it is conceivable that the Bacteria originate from the birds, rather than from the mites. That would suggest that the host range for Bartonella spp. is broader than previously reported and here we expand the list of potential sources for this zoonotic infection. However, Bartonellaceae can be symbiotic in other hosts, such as honey bees and ants [61, 62]. Further, Bartonella-like symbionts were recently found in a number astigmatid mites [63], indicating that the Bartonella detected here might be quill mite symbionts, rather than pathogens. With our data, it is not possible to rule out either possibility.

Finally, we found the symbionts Spiroplasma and Wolbachia in quill mites. Both of these are common across a range of arthropod species [41, 64], are typically transmitted intraovarially, and may cause sex-ratio distorting phenotypes [65, 66]. Whereas 
Spiroplasma was so far not reported from quill mites, Wolbachia was previously detected and our findings confirm that this is a common symbiont of quill mites [27]. The observed presence and abundance of both taxa are not uniform across the sampled taxa (Fig. 4a). For example, Wolbachia is most abundant in mites parasitizing birds of the genera Turdus, Erithacus, and Fringilla, whereas Spiroplasma is most strongly associated with mites parasitizing Carduelis. One reason for this may be that some taxa are more susceptible than others for endosymbiosis with certain Bacteria, and this phylogenetic effect has been reported for other host taxa as well [67, 68]. Strikingly, very high Spiroplasma abundances were only found in two investigated mite species that are specialised parasites of three bird species of the genus Carduelis (Figs. 1a, 4a, Supplementary Table S4). A number of samples showed very low Spiroplasma titres, which may be a result of genuine low titre infections or stem from contamination via simultaneously processed libraries (e.g., through index hopping [69]). For the samples with unambiguously high Spiroplasma titres, the bird host phylogeny seems to be the best predictor for a Spiroplasma infection. One interpretation of this pattern is a history of horizontal symbiont transmission via the bird hosts. Horizontal transfers have been inferred from phylogenetic data for Wolbachia and Spiroplasma previously [70, 71], and for both symbionts, horizontal transmissions were also demonstrated experimentally $[72,73]$. Although the potential mechanism of horizontal symbiont transmission via feather quills is unclear, our data suggest that the bird-parasite interactions may be important for endosymbiont transmission dynamics in quill mites.

Interestingly, we found that Spiroplasma presence leads to reduced Wolbachia titers, although this is based on a small sample size for samples that are both Wolbachia and Spiroplasma positive ( $\mathrm{N}=11$, Fig. 4b). Furthermore, in these eleven samples, Spiroplasma and Wolbachia titers seem to be positively correlated (Fig. 4c). It is conceivable that sharing of hosts leads to competition for finite resources the host can provide [74], and thus the growth of one symbiont might limit that of another. In Drosophila for example, Spiroplasma seem to limit the proliferation of Wolbachia [75] and in aphids, competition between co-occuring secondary symbionts appears to be harmful to the host [76]. Such negative fitness impacts can also expected when both symbiont titres are very high, as found here in quill mites. Although purely speculative, this may be the reason why we only observed simultaneously high Spiroplasma and Wolbachia titres in very few of the 126 investigated quill mites (Fig. 4c).

\section{Summary}

We find a diverse, but relatively uniform set of bacterial taxa within quill mites that includes arthropod endosymbionts, pathogens, and bird associated bacteria. The importance of most of these microbes for quill mite biology is unclear, but abundances and distribution patterns suggest that Spiroplasma and Wolbachia are the most important quill mite associates. 


\section{Acknowledgments}

We thank Dr. J. K. Nowakowski, Bird Migration Research Station, University of Gdansk for the permission to collect the material. We further thank Zuzanna

Filutowska for laboratory assistance. We are grateful to Piotr Łukasik for comments on an earlier version of this manuscript.

\section{Author contributions}

Study conception and design: EG, ZKF, MG; Collecting material EG; Acquisition of data: ZKF, MD; Data analysis: MG; Interpretation of data: EG, MG; Writing the paper EG, MG. The manuscript has been read and approved by all authors.

\section{Funding}

This study was supported by the National Science Centre of Poland (\#2015/19/D/NZ8/00191, awarded to EG). MG was supported by a Marie Sklodowska-Curie Individual Fellowship of the European Commission (H2020-MSCA-IF-2015, 703379).

\section{References}

1. McFall-Ngai, M. et al. Animals in a bacterial world, a new imperative for the life sciences. Proceedings of the National Academy of Sciences of the United States of America 110, 3229-3236 (2013).

2. Hosokawa, T., Koga, R., Kikuchi, Y., Meng, X.-Y. \& Fukatsu, T. Wolbachia as a bacteriocyte-associated nutritional mutualist. Proceedings of The National Academy of Sciences of The United States of America 107, 769-774 (2010).

3. Duron, O. et al. Tick-bacteria mutualism depends on B vitamin synthesis pathways. Current Biology 28, 1896-1902.e5 (2018).

4. Ballinger, M. \& Perlman, S. Generality of toxins in defensive symbiosis: Ribosome-inactivating proteins and defense against parasitic wasps in Drosophila. PLoS Pathogens 13, e1006431 (2017).

5. King, K. C. et al. Rapid evolution of microbe-mediated protection against pathogens in a worm host. The ISME Journal 10, 1915-1924 (2016).

6. Sharon, G. et al. Commensal bacteria play a role in mating preference of Drosophila melanogaster. Proceedings of the National Academy of Sciences of The United States of America 107, 20051-20056 (2010).

7. Laughton, A. M., Fan, M. H. \& Gerardo, N. M. The combined effects of bacterial symbionts and aging on life history traits in the pea aphid, Acyrthosiphon pisum. Applied and Environmental Microbiology 80, 470-477 (2013).

8. Hird, S. M. Evolutionary biology needs wild microbiomes. Frontiers in Microbiology 8, 725 (2017). 
9. Ji, B. \& Nielsen, J. From next-generation sequencing to systematic modeling of the gut microbiome. Frontiers in Genetics 6, 219 (2015).

10. Sboner, A., Mu, X., Greenbaum, D., Auerbach, R. K. \& Gerstein, M. B. The real cost of sequencing: higher than you think! Genome Biology 12, 125 (2011).

11. Colston, T. J. \& Jackson, C. R. Microbiome evolution along divergent branches of the vertebrate tree of life: what is known and unknown. Molecular Ecology 25, 3776-3800 (2016).

12. Erban, T. et al. Populations of stored product mite Tyrophagus putrescentiae differ in their bacterial communities. Frontiers in Microbiology 7, 1046 (2016).

13. Hubert, J., Kopecky, J., Nesvorna, M., Perotti, M. A. \& Erban, T. Detection and localization of Solitalea-like and Cardinium bacteria in three Acarus siro populations (Astigmata: Acaridae). Experimental and Applied Acarology 70, 309-327 (2016).

14. Chan, T.-F. et al. The draft genome, transcriptome, and microbiome of Dermatophagoides farinae reveal a broad spectrum of dust mite allergens. Journal of Allergy and Clinical Immunology 135, 539-548 (2015).

15. Oh, H., Ishii, A., Tongu, Y. \& Itano, K. Microorganisms associated with the house-dust mite, Dermatophagoides. Medical Entomology and Zoology 37, 229-235 (1986).

16. Valerio, C., Murray, P., Arlian, L. \& Slater, J. Bacterial 16S ribosomal DNA in house dust mite cultures. Journal of Allergy and Clinical Immunology 116, 1296-1300 (2005).

17. Hogg, J. \& Lehane, M. Identification of bacterial species associated with the sheep scab mite (Psoroptes ovis) by using amplified genes coding for $16 \mathrm{~S}$ rRNA. Applied and Environmental Microbiology 65, 4227-4229 (1999).

18. Hubert, J. et al. Comparison of microbiomes between red poultry mite populations (Dermanyssus gallinae): predominance of Bartonella-like bacteria. Microbial Ecology 74, 947-960 (2017).

19. Moro, C. V., Thioulouse, J., Chauve, C. \& Zenner, L. Diversity, geographic distribution, and habitat-specific variations of microbiota in natural populations of the chicken mite, Dermanyssus gallinae. Journal of Medical Entomology 48, 788-796 (2011).

20. Hubert, J. et al. Comparison of Varroa destructor and worker honeybee microbiota within hives indicates shared bacteria. Microbial Ecology 72, 448-459 (2016).

21. Casto, S. D. A nocturnal dispersal rhythm in the quill mite, Syringophiloidus minor (Berlese) (Prostigmata: Syringophilidae). Journal of Medical Entomology 11, 113-114 (1974).

22. Casto, S. D. Entry and exit of syringophilid mites (Acarina: Syringophilidae) from the lumen of the quill. The Wilson Bulletin 86, 272-278 (1974).

23. Kethley, J. A revision of the family Syringophilidae (Prostigmata: Acarina). Contributions of the American Entomological Institute 5, 1-76 (1970).

24. Kethley, J. Population regulation in quill mites (Acarina: Syringophilidae). Ecology 52, 1113-1118 (1971).

25. Azad, A. F. \& Beard, C. B. Rickettsial pathogens and their arthropod vectors. Emerging Infectious Diseases 4, 179 (1998).

26. Skoracki, M. et al. First detection of Anaplasma phagocytophilum in quill mites (Acari: Syringophilidae) parasitizing passerine birds. Microbes and Infection $\mathbf{8}$, 303-307 (2006). 
27. Glowska, E., Dragun-Damian, A., Dabert, M. \& Gerth, M. New Wolbachia supergroups detected in quill mites (Acari: Syringophilidae). Infection, Genetics and Evolution 30, 140-146 (2015).

28. Dabert, J., Ehrnsberger, R. \& Dabert, M. Glaucalges tytonis sp. n. (Analgoidea, Xolalgidae) from the barn owl Tyto alba (Strigiformes, Tytonidae): compiling morphology with DNA barcode data for taxon descriptions in mites (Acari). Zootaxa 1719, 41-52 (2008).

29. Skoracki, M., Spicer, G. S. \& Oconnor, B. M. A systematic review of the subfamily Syringophilinae (Acari: Syringophilidae) of the Nearctic region. Part 1: quill mites associated with passerines (Aves: Passeriformes). Zootaxa 4084, 451 (2016).

30. Martin, M. Cutadapt removes adapter sequences from high-throughput sequencing reads. EMBnet.journal 17, 10-12 (2011).

31. Callahan, B. J. et al. DADA2: High-resolution sample inference from Illumina amplicon data. Nature Methods 13, 581-583 (2016).

32. R Core Team. R: A language and environment for statistical computing (R Foundation for Statistical Computing, Vienna, Austria, 2015).

33. Quast, C. et al. The SILVA ribosomal RNA gene database project: improved data processing and web-based tools. Nucleic Acids Research 41, D590-D596 (2012).

34. Davis, N. M., Proctor, D., Holmes, S. P., Relman, D. A. \& Callahan, B. J. Simple statistical identification and removal of contaminant sequences in marker-gene and metagenomics data. bioRxiv, 221499 (2017).

35. McMurdie, P. J. \& Holmes, S. Phyloseq: an R package for reproducible interactive analysis and graphics of microbiome census data. PLoS ONE 8, e61217 (2013).

36. Wickham, H. ggplot2: elegant graphics for data analysis (Springer-Verlag New York, 2009).

37. Benjamini, Y. \& Hochberg, Y. Controlling the false discovery rate: a practical and powerful approach to multiple testing. Journal of the Royal Statistical Society Series B 57, 289-300 (1995).

38. Dixon, P. VEGAN, a package of R functions for community ecology. Journal of Vegetation Science 14, 927-930 (2003).

39. Jetz, W., Thomas, G. H., Joy, J. B., Hartmann, K. \& Mooers, A. O. The global diversity of birds in space and time. Nature 491, 444-448 (2012).

40. Kwong, W. K. et al. Dynamic microbiome evolution in social bees. Science Advances 3, e1600513 (2017).

41. Anbutsu, H. \& Fukatsu, T. Spiroplasma as a model insect endosymbiont. Environmental Microbiology Reports 3, 144-153 (2011).

42. Makepeace, B. L. \& Gill, A. C. Wolbachia in Rickettsiales. Biology, Molecular Biology, Epidemiology, and Vaccine Development (ed Thomas, S.) (Springer Nature, 2016), 465-512.

43. Hammer, T. J., Janzen, D. H., Hallwachs, W., Jaffe, S. P. \& Fierer, N. Caterpillars lack a resident gut microbiome. Proceedings of The National Academy of Sciences of The United States of America 114, 9641-9646 (2017).

44. Dille, J. W., Rogers, C. M. \& Schneegurt, M. A. Isolation and characterization of bacteria from the feathers of wild Dark-eyed Juncos (Junco hyemalis). The Auk 133, 155-167 (2016).

45. Engel, K. et al. Individual- and species-specific skin microbiomes in three different estrildid finch species revealed by $16 \mathrm{~S}$ amplicon sequencing. Microbial Ecology 76, $518-529$ (2018). 
46. Pearce, D. S., Hoover, B. A., Jennings, S., Nevitt, G. A. \& Docherty, K. M. Morphological and genetic factors shape the microbiome of a seabird species (Oceanodroma leucorhoa) more than environmental and social factors. Microbiome 5, 146 (2017).

47. Salter, S. J. et al. Reagent and laboratory contamination can critically impact sequence-based microbiome analyses. BMC Biology 12, 87 (2014).

48. Łukasik, P. et al. The structured diversity of specialized gut symbionts of the New World army ants. Molecular Ecology 26, 3808-3825 (2017).

49. De Goffau, M. C. et al. Recognizing the reagent microbiome. Nature Microbiology 3, 851-853 (2018).

50. Pappas, G., Papadimitriou, P., Akritidis, N., Christou, L. \& Tsianos, E. V. The new global map of human brucellosis. The Lancet Infectious Diseases 6, 91-99 (2006).

51. Young, E. J. Brucella spp. in Principles and practice of clinical bacteriology (eds Gillespie, S. H. \& Hawkey, P. M.) (John Wiley \& Sons, Ltd, 2006), 265-271.

52. Neglia, G. et al. Detection of Brucella abortus DNA and RNA in different stages of development of the sucking louse Haematopinus tuberculatus. BMC Veterinary Research 9, 236 (2013).

53. Pritulin, P. On the transmission of brucellosis by the pasture ticks Dermacentor nuttallia and Hyalomma marginatum. Veterinariya 31, 31-33 (1954).

54. Rementzova, M. Parasitical arthropods vectors of the brucellic infection in Proceedings of the First International Congress of Parasitology (ed Corradetti, A.) (Pergamon, 1966), 147-150.

55. Zheludkov, M. \& Tsirelson, L. Reservoirs of Brucella infection in nature. Biology Bulletin 37, 709-715 (2010).

56. Billeter, S., Levy, M., Chomel, B. \& Breitschwerdt, E. Vector transmission of Bartonella species with emphasis on the potential for tick transmission. Medical and Veterinary Entomology 22, 1-15 (2008).

57. Klangthong, K. et al. The distribution and diversity of Bartonella species in rodents and their ectoparasites across Thailand. PLoS ONE 10, e0140856 (2015).

58. Reeves, W. K., Nelder, M. P., Cobb, K. D. \& Dasch, G. A. Bartonella spp. in deer keds, Lipoptena mazamae (Diptera: Hippoboscidae), from Georgia and South Carolina, USA. Journal of Wildlife Diseases 42, 391-396 (2006).

59. Ebani, V. V., Bertelloni, F. \& Mani, P. Molecular survey on zoonotic tick-borne bacteria and chlamydiae in feral pigeons (Columba livia domestica). Asian Pacific Journal of Tropical Medicine 9, 324-327 (2016).

60. Mascarelli, P. E., McQuillan, M., Harms, C. A., Harms, R. V. \& Breitschwerdt, E. B. Bartonella henselae and B. koehlerae DNA in birds. Emerging Infectious Diseases 20, 490-492 (2014).

61. Segers, F. H., Kešnerová, L., Kosoy, M. \& Engel, P. Genomic changes associated with the evolutionary transition of an insect gut symbiont into a blood-borne pathogen. The ISME Journal 11, 1232-1244 (2017).

62. Bisch, G. et al. Genome evolution of Bartonellaceae symbionts of ants at the opposite ends of the trophic scale. Genome Biology and Evolution 10 (ed Martinez-Romero, E.) 1687-1704 (2018).

63. Kopecký, J., Nesvorná, M. \& Hubert, J. Bartonella-like bacteria carried by domestic mite species. Experimental and Applied Acarology 64, 21-32 (2014). 
64. Zug, R. \& Hammerstein, P. Still a host of hosts for Wolbachia: analysis of recent data suggests that $40 \%$ of terrestrial arthropod species are infected. PLoS ONE 7, e38544 (2012).

65. Haselkorn, T. S. The Spiroplasma heritable bacterial endosymbiont of Drosophila. Fly 4, 80-87 (2010).

66. Werren, J. H. Biology of Wolbachia. Annual Review of Entomology 42, 587-609 (1997).

67. Gerth, M., Saeed, A., White, J. A. \& Bleidorn, C. Extensive screen for bacterial endosymbionts reveals taxon-specific distribution patterns among bees (Hymenoptera, Anthophila). FEMS Microbiology Ecology 91, fiv047 (2015).

68. Russell, J. A. The ants (Hymenoptera: Formicidae) are unique and enigmatic hosts of prevalent Wolbachia (Alphaproteobacteria) symbionts. Myrmecological News 16, 7-23 (2012).

69. Kircher, M., Sawyer, S. \& Meyer, M. Double indexing overcomes inaccuracies in multiplex sequencing on the Illumina platform. Nucleic Acids Research 40, e3 (2012).

70. Gerth, M., Röthe, J. \& Bleidorn, C. Tracing horizontal Wolbachia movements among bees (Anthophila): a combined approach using MLST data and host phylogeny. Molecular Ecology 22, 6149-6162 (2013).

71. Haselkorn, T. S., Markow, T. a. \& Moran, N. A. Multiple introductions of the Spiroplasma bacterial endosymbiont into Drosophila. Molecular Ecology 18, 1294-305 (2009).

72. Huigens, M. E. et al. Infectious parthenogenesis. Nature 405, 178-179 (2000).

73. Jaenike, J., Polak, M., Fiskin, A., Helou, M. \& Minhas, M. Interspecific transmission of endosymbiotic Spiroplasma by mites. Biology Letters 3, 23-25 (2007).

74. Vautrin, E. \& Vavre, F. Interactions between vertically transmitted symbionts: cooperation or conflict? Trends in Microbiology 17, 95-99 (2009).

75. Goto, S., Anbutsu, H. \& Fukatsu, T. Asymmetrical interactions between Wolbachia and Spiroplasma endosymbionts coexisting in the same insect host. Applied and Environmental Microbiology 72, 4805-4810 (2006).

76. Oliver, K. M., Moran, N. A. \& Hunter, M. S. Costs and benefits of a superinfection of facultative symbionts in aphids. Proceedings of the Royal Society of London B: Biological Sciences 273, 1273-1280 (2006).

\section{Supporting Information}

\section{Figure S1}

Overview of the bacterial taxa detected in quill mites. a) Including the endosymbionts Wolbachia and Spiroplasma, and b) excluding these taxa. Bar plots show the 20 most abundantly found bacterial families in each dataset. Each stacked bar represents one sample, and the samples are ordered by quill mite species. Height of stacks represent relative abundances of each taxon. Note that all Anaplasmataceae ASVs are Wolbachia, and all Spiroplasmataceae ASVs are Spiroplasma. 


\section{Table S1}

Fusion PCR primers sequences used in this study. Unique random barcode sequences are highlighted in bold.

\section{Table S2}

Overview on the impact of filtering and decontamination on the number of retained ASVs and samples in this study. For details on each of the steps please refer to the Materials and methods section.

\section{Table S3}

List of all ASVs detected in this study, ordered by abundance (relative abundances summed over all samples).

\section{Table S4}

Average abundance of Spiroplasma and Wolbachia across sampled bird and mite species.

\section{Additional file 1}

Phyloseq object including all ASVs, sample and metadata information. 